\title{
46,XY Gonadal Dysgenesis
}

National Cancer Institute

\section{Source}

National Cancer Institute. 46,XY Gonadal Dysgenesis. NCI Thesaurus. Code C120198.

Gonadal dysgenesis in an individual with 46.XY karyotype. 